Ann. Funct. Anal. 6 (2015), no. 3, 145-154

http://doi.org/10.15352/afa/06-3-12

ISSN: 2008-8752 (electronic)

http://projecteuclid.org/afa

\title{
A MAX VERSION OF PERRON-FROBENIUS THEOREM FOR NONNEGATIVE TENSOR
}

\author{
HAMID REZA AFSHIN*, ALI REZA SHOJAEIFARD \\ Communicated by Q.-W. Wang
}

\begin{abstract}
In this paper we generalize the max algebra system of nonnegative matrices to the class of nonnegative tensors and derive its fundamental properties. If $\mathbb{A} \in \Re_{+}^{[m, n]}$ is a nonnegative essentially positive tensor such that satisfies the condition class $\mathrm{NC}$, we prove that there exist $\mu(\mathbb{A})$ and a corresponding positive vector $x$ such that $\max _{1 \leq i_{2} \cdots i_{m} \leq n}\left\{a_{i i_{2} \cdots i_{m}} x_{i_{2}} \cdots x_{i_{m}}\right\}=\mu(\mathbb{A}) x_{i}^{m-1}, \quad i=$ $1,2, \cdots, n$. This theorem, is well known as the max algebra version of PerronFrobenius theorem for this new system.
\end{abstract}

\section{INTRODUCTION}

The algebraic system max algebra provide an attractive way of describing a class of non-linear problems appearing for instance in manufacturing and transportation scheduling, information technology, discrete event-dynamic systems, combinatorial optimization, mathematical physics, DNA analysis and etcetera. The usefulness of max algebras arises from a fact that these non-linear problems become linear when described in the max algebra language. The max eigenproblem is well studied and there are important explicit applications of it in solving the problems mentioned above. In particular, there exists significant analogy with the usual Perron-Frobenius theory. For a recent reference focussing specifically on the Perron-Frobenius Theorem for the max algebra, see [1], wherein several proofs of this fundamental result were presented.

Tensors are increasingly ubiquitous in various areas of applied, computational, and industrial mathematics and have wide applications in data analysis and mining, information science, signal/image processing, computational biology,

Date: Received: Oct. 2, 2014; Revised: Dec. 6, 2014; Accepted: Jan. 20, 2015.

* Corresponding author.

2010 Mathematics Subject Classification. Primary 15A18; Secondary 15A69, 74B99.

Key words and phrases. Perron-Frobenius theory, max algebra, nonnegative tensor. 
and so on, see the workshop report [6] and references therein. A tensor can be regarded as a higher order generalization of a matrix, which takes the form

$$
\mathbb{A}=\left(a_{i_{1}, \cdots, i_{m}}\right), \quad a_{i_{1}, \cdots, i_{m}} \in \Re, \quad 1 \leq i_{1}, \cdots, i_{m} \leq n,
$$

where $\Re$ is a real field. Such a multi-array $\mathbb{A}$ is said to be an $m$ th order $n$ dimensional square real tensor with $n^{m}$ entries $a_{i_{1}, \cdots, i_{m}}$. In this regard, a vector is a first order tensor and a matrix is a second order tensor. Tensors of order more than two are called higher order tensors. Many important ideas, notions, and results have been successfully extended from matrices to higher order tensors. Among these, in particular, are the notions and certain basic algebraic and geometric properties of rank, eigenvalue, eigenvector, see [8]. Nonnegative tensors have attracted more and more attention because they share some intrinsic properties with those of the nonnegative matrices. One of those properties is the Perron-Frobenius theorem on eigenvalues. In [2], Chang et al. generalized the Perron-Frobenius theorem for nonnegative matrices to irreducible nonnegative tensors. In [3], Friedland et al. generalized the Perron-Frobenius theorem to weakly irreducible nonnegative tensors. Further generalization of the PerronFrobenius theorem to nonnegative tensors can be found in [10].

Now, the question arises is it possible to define max algebra system for nonnegative tensors as a generalization of max algebra on nonnegative matrices? Can we describe the analogue of the Perron-Frobenius theory for the system of max algebra on nonnegative tensors, as a generalization of max version theory which is proved in [1]?, in this paper we show the answer is affirmative.

The paper is organized as follows. In Section 2 the fundamental concept of max algebra system and tensors are given briefly for readers. In Section 3 the max algebra system is generalized to nonnegative tensors, also the new version of the Perron-Frobenius theory for some class of nonnegative tensors is given.

We first add a comment on the notation that is used. Vectors are written as $(x, y, \cdots)$, matrices correspond to $(A, B, \cdots)$ and tensors are written as $(\mathbb{A}, \mathbb{B}, \cdots)$. The entry with row index $\mathrm{i}$ and column index $\mathrm{j}$ in a matrix $A$, i.e. $(A)_{i j}$ is symbolized by $a_{i j}\left(\right.$ also $\left.(\mathbb{A})_{i_{1} i_{2} \cdots i_{m}}=a_{i_{1} i_{2} \cdots i_{m}}\right) . \Re$ and $\mathcal{C}$ represents the real and complex field, respectively. For each nonnegative integer $n$, denote $[n]=$ $\{1,2, \cdots, n\} . \Re_{+}^{n}\left(\Re_{++}^{n}\right)$ denotes the cone $\left\{x \in \Re^{n}: x_{i} \geq(>) 0, i=1, \cdots, n\right\}$.

\section{Preliminaries}

2.1. Max algebra system. In this section we give the basic definition of the max algebra. The max algebra we consider here is the set $\Re_{+}$of nonnegative real numbers, where for $a, b \in \Re_{+}$the sum $a \oplus b$ is defined as $\max \{a, b\}$ and the product is defined as the usual product $a b$. For vectors $x=\left(x_{i}\right), y=\left(y_{i}\right)$ in $\Re_{+}^{n}$ and $c \in \Re_{+}$the vectors $x \oplus y=\left(\max \left\{x_{i}, y_{i}\right\}\right)$ and $c x=\left(c x_{i}\right)$ are defined entrywise. The sum $A \oplus B$ of two matrices is defined analogously. If $A=\left(a_{i k}\right)$ is a nonnegative $n$-by- $n$ matrix then the map

$$
x \in \Re_{+} \Rightarrow A \otimes x \in \Re_{+}
$$


where $(A \otimes x)_{i}=\max _{k} a_{i k} x_{k}, i=1, \cdots, n$ is linear in the sense given above, namely for all $x, y \in \Re_{+}^{n}, c \in \Re_{+}$

$$
A \otimes(x \oplus y)=(A \otimes x) \oplus(A \otimes y), A \otimes(c x)=c(A \otimes x) .
$$

The max-product $C=\left(c_{i l}\right)=A \otimes B$ of two $n$-by- $n$ nonnegative matrices $A=\left(a_{i k}\right)$ and $B=\left(b_{k l}\right)$ is defined by $c_{i l}=\max _{k} a_{i k} b_{k l}, i, l=1,2, \cdots, n$. The weighted directed graph $G(A)$ associated with $A$ has vertex set $\{1,2, \cdots, n\}$ and an edge $(i, j)$ from vertex $i$ to vertex $j$ with weight $a_{i j}$ if and only if $a_{i j}>0$. A path $L\left(i_{1}, i_{2}, \cdots, i_{k+1}\right)$ of length $k$ is a sequence of $k$ edges

$$
\left(i_{1}, i_{2}\right),\left(i_{2}, i_{3}\right), \cdots,\left(i_{k}, i_{k+1}\right) .
$$

The weight of a path $L\left(i_{1}, i_{2}, \cdots, i_{k+1}\right)$, as denoted by $w\left(L\left(i_{1}, i_{2}, \cdots, i_{k+1}\right)\right)$ or simply by $w(L)$, is defined by

$$
w\left(L\left(i_{1}, i_{2}, \cdots, i_{k+1}\right)\right)=a_{i_{1} i_{2}} a_{i_{2} i_{3}} \cdots a_{i_{k} i_{k+1}} .
$$

A circuit $C$ of length $k$ is a path $L\left(i_{1}, i_{2}, \cdots, i_{k+1}\right)$ with $i_{k+1}=i_{1}$, where $i_{1}, i_{2}, \cdots, i_{k+1}$ are distinct. Associated with this circuit $C$ is the circuit geometric mean known as $w(C)=\left(a_{i_{1} i_{2}} a_{i_{2} i_{3}} \cdots a_{i_{k} i_{1}}\right)^{\frac{1}{k}}$. The maximum circuit geometric mean in $G(A)$ is denoted by $\mu(A)$. Note that we also consider empty circuits, namely, circuits that consist of only one vertex and have length 0 . For empty circuits, the associated circuit geometric mean is zero. In the literature, the maximum circuit geometric mean $\mu(A)$ has been studied extensively, and it is known that $\mu(A)$ is the largest max eigenvalue of $A$. Moreover, if $A$ is irreducible, then $\mu(A)$ is the unique eigenvalue and every eigenvector is positive.

Definition 2.1. [1] Let $A$ be an $n \times n$ nonnegative matrix. We say that $\lambda$ is a max eigenvalue of $A$ if there exists a nonzero, nonnegative vector $x$ such that $A \otimes x=\lambda x$. We refer to $x$ as a corresponding max eigenvector.

Theorem 2.2. Let $A$ be an $n \times n$ nonnegative, irreducible matrix. Then there exists a positive vector $x$ such that $A \otimes x=\mu(A) x$.

Proof. See [1].

2.2. Basic definition of tensor. In this subsection, we will cover some fundamental notions and properties on tensors. A tensor can be regarded as a higher order generalization of a matrix, which takes the form

$$
\mathbb{A}=\left(a_{i_{1}, \cdots, i_{m}}\right), \quad a_{i_{1}, \cdots, i_{m}} \in \Re, \quad 1 \leq i_{1}, \cdots, i_{m} \leq n,
$$

where $\Re$ is a real field. Such a multi-array $\mathbb{A}$ is said to be an $m$ th order $n$ dimensional square real tensor with $n^{m}$ entries $a_{i_{1}, \cdots, i_{m}}$. In this regard, a vector is a first order tensor and a matrix is a second order tensor. Tensors of order more than two are called higher order tensors. An $m$ th order $n$-dimensional tensor $\mathbb{A}$ is called nonnegative if $a_{i_{1} i_{2} \cdots i_{m}} \geq 0$. We denote the set of all nonnegative $m$ th order $n$-dimensional tensors by $\Re_{+}^{[m, n]}$. For a vector $x=\left(x_{1}, \cdots, x_{n}\right)^{T}$, let $\mathbb{A} x^{m-1}$ 
be a vector in $\Re^{n}$ whose $i$ th component is defined as the following:

$$
\left(\mathbb{A} x^{m-1}\right)_{i}=\sum_{i_{2}, \cdots, i_{m}=1}^{n} a_{i i_{2} \cdots i_{m}} x_{i_{2}} \cdots x_{i_{m}}
$$

and let $x^{[m]}=\left(x_{1}^{m}, \cdots, x_{n}^{m}\right)^{T}$.

Definition 2.3. [8] A pair $(\lambda, x) \in \mathcal{C} \times\left(\mathcal{C}^{n} \backslash\{0\}\right)$ is called an eigenvalue and an eigenvector of $\mathbb{A} \in \Re^{[m, n]}$, if they satisfy

$$
\mathbb{A} x^{m-1}=\lambda x^{[m-1]} .
$$

Definition 2.4. [9] Let $\mathbb{A}$ (and $\mathbb{B}$ ) be an order $m \geq 2$ (and order $k \geq 1$ ), dimension $n$ tensor, respectively. The product $\mathbb{A B}$ is defined to be the following tensor $\mathbb{C}$ of order $(m-1)(k-1)+1$ and dimension $n$ :

$$
c_{i \alpha_{1} \cdots \alpha_{m-1}}=\sum_{i_{2}, \cdots, i_{m}=1}^{n} a_{i i_{2} \cdots i_{m}} b_{i_{2} \alpha_{1}} \cdots b_{i_{m} \alpha_{m-1}},
$$

where $\left(i \in[n], \alpha_{1}, \cdots, \alpha_{m-1} \in[n]^{k-1}\right)$.

It is easy to check from the definition that $I_{n} \mathbb{A}=\mathbb{A}=\mathbb{A} I_{n}$, where $I_{n}$ is the identity matrix of order $n$. When $k=1$ and $\mathbb{B}=x \in \mathcal{C}^{n}$ is a vector of dimension $n$, then $(m-1)(k-1)+1=1$. Thus $\mathbb{A} \mathbb{B}=\mathbb{A} x$ is still a vector of dimension $n$, and we have

$$
(\mathbb{A} x)_{i}=(\mathbb{B})_{i}=c_{i}=\sum_{i_{2} \cdots i_{m}=1}^{n} a_{i i_{2} \cdots i_{m}} x_{i_{2}} \cdots x_{i_{m}}=\left(\mathbb{A} x^{m-1}\right)_{i}
$$

thus we have $\mathbb{A} x^{m-1}=\mathbb{A} x$. So the first application of the tensor product defined above is that now $\mathbb{A} x^{m-1}$ can be simply written as $\mathbb{A} x$.

Definition 2.5. [5] A tensor $\mathbb{A} \in \Re^{[m, n]}$ is called reducible, if there exists a nonempty proper index subset $I \subset\{1, \cdots, n\}$ such that

$$
a_{i_{1}, \cdots, i_{m}}=0, \forall i_{1} \in I, \quad \forall i_{2}, \cdots, i_{m} \notin I,
$$

If $\mathbb{A}$ is not reducible, then we call $\mathbb{A}$ irreducible.

The following definition was first introduced by Friedland et. al. [3].

Lemma 2.6. Let $\mathbb{A} \in \Re_{+}^{[m, n]}$ be an irreducible tensor. Then for every $1 \leq i \leq n$

$$
\sum_{i_{2} \cdots i_{m}=1}^{n} a_{i i_{2} \cdots i_{m}}>0
$$

\section{MAin RESUlts}

In this section we define the max algebra system on tensors and our interest will be in describing the analogue of the Perron-Frobenius theory for this new system, referred to as the max version of the theory. 
Definition 3.1. The max algebraic addition $(\oplus)$ and multiplication $(\otimes)$ are defined as follows:

(i) Suppose that $\mathbb{A}, \mathbb{B} \in \Re_{+}^{[m, n]}$ then we have $\mathbb{A} \oplus \mathbb{B} \in \Re_{+}^{[m, n]}$ and

$$
(\mathbb{A} \oplus \mathbb{B})_{i_{1} \cdots i_{m}}=a_{i_{1} \cdots i_{m}} \oplus b_{i_{1} \cdots i_{m}}=\max \left(a_{i_{1} \cdots i_{m}}, b_{i_{1} \cdots i_{m}}\right) .
$$

(ii) Suppose that $\mathbb{A} \in \Re_{+}^{[m, n]}$ and $\mathbb{B} \in \Re_{+}^{[k, n]}$ where $m \geq 2, k \geq 1$ then we have $\mathbb{A} \otimes \mathbb{B} \in \Re_{+}^{[(m-1)(k-1)+1, n]}$ and

$$
\begin{aligned}
(\mathbb{A} \otimes \mathbb{B})_{i \alpha_{1} \cdots \alpha_{m-1}} & =\bigoplus_{i_{2} \cdots i_{m}=1}^{n} a_{i i_{2} \cdots i_{m}} b_{i_{2} \alpha_{1}} \cdots b_{i_{m} \alpha_{m-1}} \\
& =\max _{1 \leq i_{2} \cdots i_{m} \leq n}\left\{a_{i i_{2} \cdots i_{m}} b_{i_{2} \alpha_{1}} \cdots b_{i_{m} \alpha_{m-1}}\right\},
\end{aligned}
$$

where $i \in\{1, \cdots, n\}, \alpha_{1}, \cdots, \alpha_{m-1} \in[n]^{k-1}$. In particular for $x \in \Re_{+}^{n}$ we have

$$
(\mathbb{A} \otimes x)_{i}=\max _{1 \leq i_{2} \cdots i_{m} \leq n}\left\{a_{i i_{2} \cdots i_{m}} x_{i_{2}} \cdots x_{i_{m}}\right\} .
$$

Example 3.2. Let $\mathbb{A}$ and $\mathbb{B}$ be third-order two-dimensional tensors of the following form:

$$
\begin{array}{llll}
a_{111}=1 & a_{121}=2 & a_{112}=1 & a_{122}=2 \\
a_{211}=2 & a_{221}=1 & a_{212}=2 & a_{222}=1 \\
b_{111}=2 & b_{121}=0 & b_{112}=4 & b_{122}=1 \\
b_{211}=0 & b_{221}=3 & b_{212}=1 & b_{222}=0
\end{array}
$$

if $\mathbb{C}=\mathbb{A} \otimes \mathbb{B}$, then for example $c_{12112}=24$.

If $x=\left(\begin{array}{l}4 \\ 5\end{array}\right)$ then $(\mathbb{A} \otimes x)=\left(\begin{array}{l}50 \\ 40\end{array}\right)$.

Theorem 3.3. The max algebraic addition $(\oplus)$ and multiplication $(\otimes)$ have the following properties:

(i) Let $\mathbb{A} \in \Re_{+}^{[m, n]}$ then $I_{n} \otimes \mathbb{A}=\mathbb{A}=\mathbb{A} \otimes I_{n}$ where $I_{n}$ is a identity matrix.

(ii) $(\lambda \mathbb{A}) \otimes \mathbb{B}=\lambda(\mathbb{A} \otimes \mathbb{B})$ where $\lambda$ be a nonnegative number.

(iii) $\mathbb{A} \otimes(\lambda \mathbb{B})=\lambda^{m-1}(\mathbb{A} \otimes \mathbb{B})$ where $\lambda$ be a nonnegative number.

(iv) Let $\mathbb{A}_{1}, \mathbb{A}_{2} \in \Re_{+}^{[m, n]}$ and $\mathbb{B} \in \Re_{+}^{[k, n]}$ then $\left(\mathbb{A}_{1} \oplus \mathbb{A}_{2}\right) \otimes \mathbb{B}=\left(\mathbb{A}_{1} \otimes \mathbb{B}\right) \oplus\left(\mathbb{A}_{2} \otimes \mathbb{B}\right)$. (v) Let $A$ be an $n \times n$ matrix and $\mathbb{B}_{1}, \mathbb{B}_{2} \in \Re_{+}^{[k, n]}$ then $A \otimes\left(\mathbb{B}_{1} \oplus \mathbb{B}_{2}\right)=\left(A \otimes \mathbb{B}_{1}\right) \oplus$ $\left(A \otimes \mathbb{B}_{2}\right)$. (Note that in general when $A$ is not a matrix, then the right distributivity doesn't hold.)

Proof. The proof of (i), (ii) and (iii) is trivial. We also have

$$
\begin{aligned}
& \left(\left(\mathbb{A}_{1} \oplus \mathbb{A}_{2}\right) \otimes \mathbb{B}\right)_{i \alpha_{1} \cdots \alpha_{m-1}}=\max _{1 \leq i_{2}, \cdots, i_{m} \leq n}\left(\left(\mathbb{A}_{1} \oplus \mathbb{A}_{2}\right)_{i i_{2} \cdots i_{m}} b_{i_{2} \alpha_{1}} \cdots b_{i_{m} \alpha_{m-1}}\right) \\
& =\max _{1 \leq i_{2}, \cdots, i_{m} \leq n}\left(\max \left(\left(\mathbb{A}_{1}\right)_{i i_{2} \cdots i_{m}},\left(\mathbb{A}_{2}\right)_{i i_{2} \cdots i_{m}}\right) b_{i_{2} \alpha_{1}} \cdots b_{i_{m} \alpha_{m-1}}\right) \\
& =\max _{1 \leq i_{2}, \cdots, i_{m} \leq n}\left(\max \left(\left(\mathbb{A}_{1}\right)_{i i_{2} \cdots i_{m}} b_{i_{2} \alpha_{1}} \cdots b_{i_{m} \alpha_{m-1}},\left(A_{2}\right)_{i i_{2} \cdots i_{m}} b_{i_{2} \alpha_{1}} \cdots b_{i_{m} \alpha_{m-1}}\right)\right) \\
& =\max \left(\max _{1 \leq i_{2}, \cdots, i_{m} \leq n}\left(\left(\mathbb{A}_{1}\right)_{i i_{2} \cdots i_{m}} b_{i_{2} \alpha_{1}} \cdots b_{i_{m} \alpha_{m-1}},\left(\mathbb{A}_{2}\right)_{i i_{2} \cdots i_{m}} b_{i_{2} \alpha_{1}} \cdots b_{i_{m} \alpha_{m-1}}\right)\right) \\
& =\max \left(\max _{1 \leq i_{2}, \cdots, i_{m} \leq n}\left(\mathbb{A}_{1}\right)_{i i_{2} \cdots i_{m}} b_{i_{2} \alpha_{1}} \cdots b_{i_{m} \alpha_{m-1}}, \max _{1 \leq i_{2}, \cdots, i_{m} \leq n}\left(\mathbb{A}_{2}\right)_{i i_{2} \cdots i_{m}} b_{i_{2} \alpha_{1}} \cdots b_{i_{m} \alpha_{m-1}}\right) \\
& =\left(\left(\mathbb{A}_{1} \otimes \mathbb{B}\right) \oplus\left(\mathbb{A}_{2} \otimes \mathbb{B}\right)\right)_{i \alpha_{1} \cdots \alpha_{m-1}} .
\end{aligned}
$$

Thus the proof of (iv) is complete. The proof of (v) is similar. 
Now we use a method similar with the proof of Theorem 1.1 in [9] to show the associative law.

Theorem 3.4. Let $\mathbb{A}$ (and $\mathbb{B}, \mathbb{C}$ ) be an order $m+1$ (and order $k+1$, order $r+1)$, dimension $n$ tensor, respectively. Then we have

$$
\mathbb{A} \otimes(\mathbb{B} \otimes \mathbb{C})=(\mathbb{A} \otimes \mathbb{B}) \otimes \mathbb{C} .
$$

Proof. For $\beta_{1}, \cdots, \beta_{m} \in\left([n]^{r}\right)^{k}$, we write:

$\beta_{1}=\theta_{11} \cdots \theta_{1 k}, \cdots, \beta_{m}=\theta_{m 1} \cdots \theta_{m k} \quad\left(\theta_{i j} \in[n]^{r}, i=1, \cdots, m ; j=1, \cdots, k\right)$.

Then we have:

$$
\begin{gathered}
(\mathbb{A} \otimes(\mathbb{B} \otimes \mathbb{C}))_{i \beta_{1} \cdots \beta_{m}}=\max _{1 \leq i_{1}, \cdots, i_{m} \leq n} a_{i i_{1} \cdots i_{m}}\left(\prod_{j=1}^{m}(\mathbb{B} \otimes \mathbb{C})_{i_{j} \beta_{j}}\right) \\
=\max _{1 \leq i_{1}, \cdots, i_{m} \leq n} a_{i i_{1} \cdots i_{m}}\left(\prod_{j=1}^{m}(\mathbb{B} \otimes \mathbb{C})_{i_{j} \theta_{j 1} \cdots \theta_{j k}}\right) \\
=\max _{1 \leq i_{1}, \cdots, i_{m} \leq n} a_{i i_{1} \cdots i_{m}}\left(\prod_{j=1}^{m} \max _{1 \leq t_{j 1}, \cdots, t_{j k} \leq n} b_{i_{j} t_{j 1} \cdots t_{j k}}\left(c_{t_{j 1} \theta_{j 1}} \cdots c_{t_{j k} \theta_{j k}}\right)\right) \\
=\max _{1 \leq i_{1}, \cdots, i_{m} \leq n} a_{i i_{1} \cdots i_{m}} \max _{1 \leq t_{j h} \leq n(1 \leq j \leq m ; 1 \leq h \leq k)}\left(\prod_{j=1}^{m} b_{i_{j} t_{j 1} \cdots t_{j k}}\left(c_{t_{j 1} \theta_{j 1}} \cdots c_{t_{j k} \theta_{j k}}\right)\right) .
\end{gathered}
$$

On the other hand, for $\alpha_{1}, \cdots, \alpha_{m} \in[n]^{k}$, we write:

$$
\alpha_{1}=t_{11} \cdots t_{1 k}, \cdots, \alpha_{m}=t_{m 1} \cdots t_{m k} \quad\left(t_{i j} \in[n], i=1, \cdots, m ; j=1 \cdots, k\right) .
$$

Then we also have:

$$
\begin{aligned}
& ((\mathbb{A} \otimes \mathbb{B}) \otimes \mathbb{C})_{i \beta_{1} \cdots \beta_{m}}=\max _{\alpha_{1}, \cdots, \alpha_{m} \in[n]^{k}}(\mathbb{A} \otimes \mathbb{B})_{i \alpha_{1} \cdots \alpha_{m}}\left(\prod_{j=1}^{n}\left(c_{t_{j 1} \theta_{j 1}} \cdots c_{t_{j k} \theta_{j k}}\right)\right) \\
& =\max _{1 \leq t_{j h} \leq n(1 \leq j \leq m ; 1 \leq h \leq k)} \max _{1 \leq i_{1}, \cdots, i_{m} \leq n} a_{i i_{1} \cdots i_{m}}\left(\prod_{j=1}^{m} b_{i_{j} \alpha_{j}}\right)\left(\prod_{j=1}^{m} c_{t_{j 1} \theta_{j 1}} \cdots c_{t_{j k} \theta_{j k}}\right) \\
& =\max _{1 \leq i_{1}, \cdots, i_{m} \leq n} a_{i i_{1} \cdots i_{m}} \max _{1 \leq t_{j h} \leq n(1 \leq j \leq m ; 1 \leq h \leq k)}\left(\prod_{j=1}^{m} b_{i_{j} t_{j 1} \cdots t_{j k}}\left(c_{t_{j 1} \theta_{j 1}} \cdots c_{t_{j k} \theta_{j k}}\right)\right) .
\end{aligned}
$$

Thus the proof is complete.

Theorem 3.5. Let $\mathbb{A}, \mathbb{B}, \in \Re_{+}^{[m, n]}$ and $T, S$ are both matrices. Then

$$
T \otimes(\mathbb{A} \oplus \mathbb{B}) \otimes S=(T \otimes \mathbb{A} \otimes S) \oplus(T \otimes \mathbb{B} \otimes S) .
$$

Proof. By the left distributive law and right distributive, the proof is clear.

Lemma 3.6. Let $\mathbb{A}, \mathbb{B}, \in \Re_{+}^{[m, n]}$ and $y \in \Re^{n}$. Then

(i) $(\mathbb{A} \oplus \mathbb{B}) \otimes y=(\mathbb{A} \otimes y) \oplus(\mathbb{B} \otimes y)$.

(ii) $\mathbb{I} \otimes y=\mathbb{I} y=y^{[m-1]}$.

Proof. By Definition 3.1 the assertion is clear. 
Lemma 3.7. Suppose that $\mathbb{A} \in \Re_{+}^{[m, n]}$ and $P$ be an $n \times n$ permutation matrix. Then

$$
P \mathbb{A} P^{T}=P \otimes \mathbb{A} \otimes P^{T}
$$

Proof. Let $\sigma \in S_{n}$ be a permutation on the set $\{1, \cdots, n\}, P=P_{\sigma}=\left(p_{i j}\right)$ be the corresponding permutation matrix of $\sigma$ where $p_{i j}=1$ iff $\sigma(i)=j$. Then

$$
(P \mathbb{A})_{i_{1} \cdots i_{m}}=a_{\sigma\left(i_{1}\right) i_{2} \cdots i_{m}}=(P \otimes \mathbb{A})_{i_{1} \cdots i_{m}}=\max _{j_{1}} a_{j_{1} i_{2} \cdots i_{m}} p_{i_{1} j_{1}} .
$$

Similarly $\mathbb{A} P=\mathbb{A} \otimes P$. Thus by Theorem 3.4 we have

$$
P \otimes \mathbb{A} \otimes P^{T}=(P \otimes \mathbb{A}) \otimes P^{T}=(P \mathbb{A}) \otimes P^{T}=P \mathbb{A} P^{T}
$$

Lemma 3.8. Let $\mathbb{A} \in \Re^{[m, n]}$ be a reducible and $P$ be an $n \times n$ permutation matrix. Then $P \mathbb{A} P^{T} \in \Re^{[m, n]}$ is a reducible tensor.

Proof.

$$
\begin{aligned}
\left(P \mathbb{A} P^{T}\right)_{i_{1} \cdots i_{m}} & =\sum_{j_{1}, \cdots, j_{m}=1}^{n} a_{j_{1} \cdots j_{m}} p_{i_{1} j_{1}}\left(\left(P^{T}\right)_{j_{2} i_{2}} \cdots\left(P^{T}\right)_{j_{m} i_{m}}\right) \\
& =\sum_{j_{1}, \cdots, j_{m}=1}^{n} a_{j_{1} \cdots j_{m}} p_{i_{1} j_{1}} p_{i_{2} j_{2}} \cdots p_{i_{m} j_{m}}=a_{\sigma\left(i_{1}\right) \cdots \sigma\left(i_{m}\right)} .
\end{aligned}
$$

Where $\sigma$ is defined in pervious lemma. Therefore by Definition 2.5 the proof is complete.

Theorem 3.9. Suppose that $\mathbb{A} \in \Re_{+}^{[m, n]}, n \geq 2$, irreducible, and $y$ is a nonnegative $n$-tuple with exactly $k$ positive coordinates, $1 \leq k \leq n-1$, then $(\mathbb{I} \oplus \mathbb{A}) \otimes y$ has more than $k$ positive coordinates.

Proof. Suppose that $k$ coordinates of $y$ are positive and the others are zero. Let $P$ be a permutation matrix such that the first $k$ coordinates of $x=P \otimes y$ are positive and the others are zero. Since $\mathbb{A}$ is a nonnegative tensor hence the number of zero coordinates in $(\mathbb{I} \oplus \mathbb{A}) \otimes y$ cannot be greater than $n-k$. Because if $y_{i}>0$ then

$$
((\mathbb{I} \oplus \mathbb{A}) \otimes y)_{i}=((\mathbb{I} \otimes y) \oplus(\mathbb{A} \otimes y))_{i}=\max _{i}\left\{y_{i}^{m-1},(\mathbb{A} \otimes y)_{i}\right\} \geq y_{i}^{m-1}>0 .
$$

Suppose it is $n-k$. That is if $y_{i}=0$ then

$$
0=((\mathbb{I} \oplus \mathbb{A}) \otimes y)_{i}=\max _{i}\left\{y_{i}^{m-1},(\mathbb{A} \otimes y)_{i}\right\}=(\mathbb{A} \otimes y)_{i} .
$$

Therefore $(P \otimes y)_{i}=0$ then $(P \otimes(\mathbb{A} \otimes y))_{i}=0$. But $x=P \otimes y$ and therefore the assumption that $(\mathbb{I} \oplus \mathbb{A}) \otimes y$ has as many 0's as $y$ is equivalent to the assertion that $\left(\left(P \otimes \mathbb{A} \otimes P^{T}\right) \otimes x\right)_{i}=0$ for $i=k+1, k+2, \cdots, n$. By relation 3.3 $\left(\left(P \mathbb{A} P^{T}\right) \otimes x\right)_{i}=0$ for $i=k+1, k+2, \cdots, n$. Let $\mathbb{B}=\left(b_{i_{1} \cdots i_{m}}\right)=P \mathbb{A} P^{T}$ Then

$$
0=(\mathbb{B} \otimes x)_{i}=\max _{1 \leq i_{2}, \cdots, i_{m} \leq n}\left\{b_{i i_{2} \cdots i_{m}} y_{i_{2}} \cdots y_{i_{m}}\right\},
$$

for $i=k+1, k+2, \cdots, n$. Therefore $\max _{1 \leq i_{2}, \cdots, i_{m} \leq k}\left\{b_{i i_{2} \cdots i_{m}} y_{i_{2}} \cdots y_{i_{m}}\right\}=0$ for $i=$ $k+1, k+2, \cdots, n$. Since for $i_{2}, \cdots, i_{m} \in\{1, \cdots, k\}$ we have $y_{i_{2}}, \cdots, y_{i_{m}}$ are positive thus $b_{i i_{2} \cdots i_{m}}=0$ for $i \in\{k+1, \cdots, n\}$ and $i_{2}, \cdots, i_{m} \in\{1, \cdots, k\}$. 
This means that $\mathbb{B}$ is reducible thus by Lemma $3.8 \mathbb{A}$ is reducible. and this is a contradiction.

Definition 3.10. Let $\mathbb{A} \in \Re_{+}^{[m, n]}$. We say that $\lambda$ is a max eigenvalue of $\mathbb{A}$ if there exists a nonzero, nonnegative vector $x$ such that $\mathbb{A} \otimes x=\lambda x^{[m-1]}$. We refer to $x$ as a corresponding max eigenvector.

Definition 3.11. For a given $\mathbb{A}=\left(a_{i_{1} \cdots i_{m}}\right) \in \Re_{+}^{[m, n]}$, it is associated to a directed graph $G(\mathbb{A})=(V, E(\mathbb{A}))$, where $V=\{1,2, \cdots, n\}$ and a directed edge $(i, j) \in$ $E(\mathbb{A})$ if there exists indices $\left\{i_{2}, \cdots, i_{m}\right\}$ such that $j \in\left\{i_{2}, \cdots, i_{m}\right\}$ and $a_{i i_{2} \cdots i_{m}}>$ 0. In particular, we have $\sum_{j \in\left\{i_{2}, \cdots, i_{m}\right\}} a_{i i_{2} \cdots i_{m}}>0$. A graph is strongly connected if it contains a directed path from $i$ to $j$ and a directed path from $j$ to $i$ for every pair of vertices $i, j$.

Definition 3.12. ([7]) Suppose that $\mathbb{A}$ is a nonnegative tensor of order $m$ and dimension $n$. $\mathbb{A}$ is called essentially positive if $\mathbb{A} x \in \Re_{++}^{n}$ for any nonzero $x \in \Re_{+}^{n}$.

It is clear that $\mathbb{A}$ is essentially positive iff for any $i, j \in[n], a_{i j \cdots j}>0$ holds. Also a nonnegative essentially positive tensor is irreducible (see Theorem (3.2) in $[7])$.

Definition 3.13. Let $\mathbb{A} \in \Re_{+}^{[m, n]}$ be an essentially positive tensor. Consider the directed graph $G(\mathbb{A})=(V, E(\mathbb{A}))$. In this directed graph, $k$ is a simple cycle of length $q$ described by a sequence of distinct integers $i_{1}, \cdots, i_{q} \in\{1, \cdots, n\}$. Then with $|k|=q$,

$$
\mu(\mathbb{A})=\max _{k}\left\{\left(a_{i_{1} i_{2} \cdots i_{2}} a_{i_{2} i_{3} \cdots i_{3}} \cdots a_{i_{q} i_{1} \cdots i_{1}}\right)^{\frac{1}{|k|}}\right\} .
$$

The following result plays a central role in the proof of the main result of this section.

Lemma 3.14. Let $\mathbb{A} \in \Re_{+}^{[m, n]}$ be irreducible tensor and $x \in \Re^{n}, x \geq 0, x \neq 0$, $\lambda>0$ such that $\mathbb{A} \otimes x=\lambda x^{[m-1]}$. Then $x$ is positive.

Proof. Suppose that $\mathbb{A} \otimes x=\lambda x^{[m-1]}$ where $\mathbb{A} \geq 0$ is irreducible, $x \geq 0$, and $x \neq 0$. Clearly, $\lambda$ must be nonnegative. Now

$$
(\mathbb{I} \oplus \mathbb{A}) \otimes x=(\mathbb{I} \otimes x) \oplus(\mathbb{A} \otimes x)=x^{[m-1]} \oplus(\mathbb{A} \otimes x)=(1 \oplus \lambda) x^{[m-1]},
$$

thus $(\mathbb{I} \oplus \mathbb{A}) \otimes x=(1 \oplus \lambda) x^{[m-1]}$. If $x$ had $k$ zero coordinates, $1 \leq k<n$, then $(1 \oplus \lambda) x^{[m-1]}$ would have $k$ zeros as well, whereas by Theorem 3.9, $(\mathbb{I} \oplus \mathbb{A}) \otimes x$ would have less than $k$ zeros. Hence $x$ must be positive.

Definition 3.15. We define $\mathrm{NC}$ to be the set of all $\mathbb{A} \in \Re_{+}^{[m, n]}$ such that for it, there exist $x \neq 0, x \in \Re_{+}^{n}$ and $\lambda>0$ such that $\mathbb{A} \otimes x=\lambda x^{[m-1]}$ and $\left\{(i, j): a_{i j \ldots j} x_{j}^{m-1}=\lambda x_{i}^{m-1}, 1 \leq i, j \leq n\right\}$ has at least a circuit.

Lemma 3.16. Let $\mathbb{A}$ be essentially positive tensor such that belong to $N C$, Then $\lambda=\mu(\mathbb{A})$. 
Proof. Since $\mathbb{A}$ is an essentially positive tensor thus it is irreducible and therefore by lemma 3.14, $x>0$. We then have

$$
(\mathbb{A} \otimes x)_{i}=\max _{1 \leq i_{2} \cdots i_{m} \leq n}\left\{a_{i i_{2} \cdots i_{m}} x_{i_{2}} \cdots x_{i_{m}}\right\}=\lambda x_{i}^{m-1}, \quad i=1,2, \cdots, n .
$$

If $\left(i_{1}, i_{2}\right),\left(i_{2}, i_{3}\right), \cdots,\left(i_{k}, i_{1}\right)$ is a circuit in $G(\mathbb{A})$, then by $(3.4)$,

$$
a_{i_{t} i_{t+1} \cdots i_{t+1}} x_{i_{t+1}}^{m-1} \leq \lambda x_{i_{t}}^{m-1}, \quad t=1,2, \cdots, k
$$

where $k+1$ is taken to be 1 . It follows that $\sqrt[k]{a_{i_{1} i_{2} \cdots i_{2}} a_{i_{2} i_{3} \cdots i_{3}} \cdots a_{i_{k} i_{1} \cdots i_{1}}} \leq \lambda$, and thus we have shown that $\mu(\mathbb{A}) \leq \lambda$ because the circuit is arbitrary. Since $\mathbb{A}$ belong to $\mathrm{NC}$, thus we conclude that $\lambda \leq \mu(\mathbb{A})$. therefore the proof is complete.

Lemma 3.17. Let $\mathbb{A} \in \Re_{+}^{[m, n]}$ be essentially positive tensor and suppose $x \in \Re^{n}$, $x \geq 0, x \neq 0, \lambda>0$ such that $\mathbb{A} \otimes x=\lambda x^{[m-1]}$. Then $\mu(\mathbb{A}) \leq \lambda$.

The following result, extends the Perron-Frobenius theorem to essentially positive nonnegative tensors over the max algebra.

Theorem 3.18. Let $\mathbb{A}$ be essentially positive tensor such that belong to $N C$. Then there exists a positive vector $x$ such that $\mathbb{A} \otimes x=\mu(\mathbb{A}) x^{[m-1]}$.

Proof. Suppose that $E=\left\{x \in \Re^{n}: x \geq 0,\|x\|_{1}=1\right\}$ which is a nonempty, compact, and convex set. Now we define the map $f: E \rightarrow E$ as

$$
f(x)=\frac{(\mathbb{A} \otimes x)^{\frac{1}{m-1}}}{\left(\sum_{i=1}^{n}(\mathbb{A} \otimes x)_{i}\right)^{\frac{1}{m-1}}}
$$

if $x \in E$. Since $\mathbb{A}$ is irreducible thus by Lemma 2.6, $f(x)$ well defined on E. Also $f$ is continuous. By Brouwer's fixed-point theorem, there exists $x_{0} \in E$ such that $f\left(x_{0}\right)=x_{0}$. Therefore

$$
\left(\mathbb{A} \otimes x_{0}\right)^{\frac{1}{m-1}}=\left(\sum_{i=1}^{n}\left(\mathbb{A} \otimes x_{0}\right)_{i}\right)^{\frac{1}{m-1}} x_{0}
$$

hence

$$
\left(\mathbb{A} \otimes x_{0}\right)=\left(\sum_{i=1}^{n}\left(\mathbb{A} \otimes x_{0}\right)_{i}\right) x_{0}{ }^{[m-1]} .
$$

By Lemma 3.14 and Lemma 3.16 we have $\mu(\mathbb{A})=\left(\sum_{i=1}^{n}\left(\mathbb{A} \otimes x_{0}\right)_{i}\right)$ and $x_{0}>0$ thus the proof is complete.

Example 3.19. Consider the positive order 3, dimension 2 tensor given by $a_{122}=$ $a_{211}=t>0, a_{i j k}=z>0$ otherwise, and let $t>z$. Then $\lambda=t$ and $x_{1}=x_{2}$ satisfy in $\mathbb{A} \otimes x=\lambda x^{[2]}$, thus we have $\mu(\mathbb{A})=t$.

Example 3.20. Consider the positive order 3, dimension 2 tensor given by $a_{121}=$ $a_{221}=1$ and $a_{i j k}=0.1$ otherwise. Then $\lambda=1$ and $x_{1}=x_{2}$ satisfy in $\mathbb{A} \otimes x=\lambda x^{[2]}$, but we have $\mu(\mathbb{A})=0.1$. 
Remark 3.21. By Theorem 2 in [4], if the function $f: \Re_{+}^{n} \rightarrow \Re_{+}^{n}$ is homogeneous, monotone and $G(A)$ is strongly connected then $f$ has an eigenvector in $\Re_{+}^{n}$. In case $m=2$ Theorem 3.18 is a result of this theorem.

Theorem 3.18 is right for the class of weakly positive tensors which satisfy in definition $\mathrm{NC}$, since for a tensor $\mathbb{A}$ belong to this class we have $a_{i j \ldots j}$ is positive for all $i \neq j$. Now it is very nice if one could generalize Theorem 3.18 to other

classes of tensors, it is an unsolved problem.

\section{REFERENCES}

1. R. Bapat, A max version of the Perron-Frobenius theorem, Proceedings of the Sixth Conference of the International Linear Algebra Society (Chemnitz, 1996). Linear Algebra Appl. 275/276 (1998), 3-18.

2. K.C. Chang, K. Pearson and T. Zhang, Perron-Frobenius theorem for nonnegative tensors, Commun. Math. Sci 6 (2008), no. 2, 507-520.

3. S. Friedland, S. Gaubert and L. Han, Perron-Frobenius theorem for nonnegative multilinear forms and extensions, Linear Algebra Appl 438 (2013), 738-749.

4. S. Gaubert and J. Gunawardena, The Perron-Frobenius theorem for homogeneous, monotone functions, Trans. Amer. Math. Soc 356 (2004), 4931-4950.

5. L.H. Lim, Singular values and eigenvalues of tensors, a variational approach, in: Proceedings 1st IEEE International Workshop on Computational Advances of Multitensor Adaptive Processing (CAMSAP '05), 1 (2005), 129-132.

6. V. Loan, Future directions in tensor based computation and modeling, NSF Workshop Report in Arlington, Virginia, USA, 2009. (Available at http://www.cs.cornell.edu/cv/TenWork/Home.htm).

7. K. Pearson, Essentially positive tensors, Int. J. Algebra 4 (2010), no. 9-12, 421-427.

8. L. Qi, Eigenvalues of a real supersymmetric tensor. J. Symbolic Comput 40 (2005), 13021324 .

9. J.Y. Shao, A general product of tensors with applications, Linear Algebra Appl 439 (2013), 2350-2366.

10. Y. Yang and Q. Yang, Further results for Perron-Frobenius theorem for nonnegative tensors, SIAM J. Matrix Anal. Appl 31 (2010), 2517-2530.

Department of Mathematics, Faculty of Mathematical Sciences, Vali-e-Asr University of RAFSANJAN, RAFSANJAN, Islamic REPUblic of Iran.

E-mail address: afshin@vru.ac.ir; alireza.shojaei@yahoo.com 